\title{
Variables predictivas del alta terapéutica entre pacientes con patologia dual grave atendidos en una comunidad terapéutica de drogodependencias con unidad psiquiátrica
}

\section{Predictors of the therapeutic discharge in patients with dual pathology admitted to a therapeutic community with a psychiatric unit}

\author{
Agustín Madoz-Gúrpide*, Vicente García Vicent**, Encarnación Luque Fuentes ***, \\ EnRIQUeta Ochoa Mangado $* * * *$
}

*CSM San Blas. Hospital Universitario Ramón y Cajal. Madrid, **CSM Las Albarizas. Marbella, Málaga, ***Asociación ACEPSA. San Pedro Alcántara, Málaga, **** Servicio de Psiquiatría. Hospital Universitario Ramón y Cajal. Madrid.

\section{Resumen}

Este trabajo tiene como objetivo analizar las variables de las que depende el alta terapéutica en los pacientes con patología dual grave (PDG) ingresados en una comunidad terapéutica profesional (CTP) donde se realiza el tratamiento integrado de su patología. 325 pacientes ingresaron entre junio de 2000 y junio de 2009 en la CTP. Se trata de un estudio retrospectivo y transversal sin grupo control, basado en el análisis detallado de la información recogida en un modelo de entrevista clínica semi-estructurada diseñado en la CTP. El 29,5\% de la muestra recibió alta terapéutica. De todas las variables estudiadas han resultado significativas el género, la edad al inicio del tratamiento, el nivel de estudios del paciente, la dependencia de opiáceos, el "policonsumo", la presencia de trastornos psicóticos y la de trastorno límite de personalidad. En nuestro estudio, el género condiciona el tipo de alta, encontrándose mayores porcentajes de alta terapéutica para las mujeres. El mayor nivel de estudios incrementa también el mejor pronóstico entendido como mayor frecuencia de alta terapéutica en individuos con estudios superiores. La edad más tardía al inicio del tratamiento disminuye la probabilidad de alta terapéutica. Del mismo modo, el policonsumo, el diagnóstico de trastornos psicóticos y de trastorno límite de personalidad se relacionan con peores resultados obteniéndose menores porcentajes de altas terapéuticas. Reconocer estas características permitirá la identificación precoz de los pacientes que tienen más riesgo de abandonar precipitadamente el tratamiento, para tratar de prevenirlo aumentando la intensidad terapéutica.

\begin{abstract}
This study aims to analyze the variables on which depends therapeutic discharge, in patients with a severe dual diagnosis admitted to a professional therapeutic community where their pathology is treated. 325 patients admitted between June 2000 and June 2009 to the therapeutic community. This is a retrospective, cross-sectional study with no control group, based on the detailed analysis of the information collected in a model of semi-structured clinical interview designed in the therapeutic community. The $29.5 \%$ of the individuals included in the sample were therapeutically discharged. Of all the variables introduced in this analysis the most significant ones were gender, age at the beginning of treatment, education level, opiate dependence, polidrug abuse, and the presence of psychotic disorders and borderline personality disorder. In our study, gender determines the type of discharge, being therapeutic discharge more frequent among women. A higher educational also increases a better prognosis with a higher rate of therapeutic discharge among individuals with higher education level. A later age at the beginning of the treatment reduces the likelihood of therapeutic discharge. Likewise, polidrug abuse, diagnosis of psychotic disorders and borderline personality disorder are associated to a lower rate of therapeutic discharge. Recognizing these characteristics will allow the early identification of those patients more at risk of dropping treatment hastily, while trying to prevent it by increasing the therapeutic intensity.
\end{abstract}

Key words: abuse drugs, severe mental disorder, severe dual diagnosis, therapeutic community, therapeutic discharge.
Palabras clave: abuso de sustancias, trastorno mental grave, patología dual grave, comunidad terapéutica profesional, alta terapéutica. 


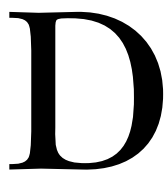

esde la primera gran encuesta sobre comorbilidad entre trastornos psiquiátricos y consumo de sustancias en población general en EEUU (Regier et al., 1990) se han publicado numerosos estudios sobre lo que ahora se conoce como patología dual (PD) en diferentes recursos de las redes asistenciales, tanto las dedicadas a la salud mental como las que se ocupan de la asistencia a adictos a sustancias de abuso. La elevada prevalencia de psicopatología en individuos con trastornos por uso de sustancias (TUS) y a la inversa, de drogodependencias asociadas en pacientes con trastorno mental grave (TMG), ya está bien descrita (Galbaud, Newman y Bland, 1993; Chambers, Cristal y Self, 2001; Sobradiel y García-Vicent, 2007; Kessler et al., 1994; Compton, Thomas, Stinson y Grant, 2007). Sin embargo, no existen muchos estudios que evalúen sistemáticamente la comorbilidad psiquiátrica en los usuarios de las comunidades terapéuticas españolas destinadas al tratamiento de pacientes con TUS, aunque la evidencia clínica refleja una alta prevalencia de trastornos psiquiátricos concurrentes, que se asocian a peores resultados en los tratamientos por abuso de drogas, especialmente en el caso de que presenten patología dual grave (PDG).

En cuanto al concepto de enfermedad mental grave incluido en la PDG, se ha considerado las pautas que define el Sistema Andaluz de Salud (SAS) en su mapa de procesos asistenciales (Consejería de Salud, 2006). Según esta clasificación "quedan incluidas en la definición del trastorno mental grave (TMG), una serie de categorías diagnósticas del CIE-10 que presentan una sintomatología de características psicóticas y/o prepsicóticas, con repercusión en distintas áreas del funcionamiento personal, una evolución prolongada en el tiempo, e importantes necesidades de continuidad de atención, coordinación interna y externa y un abordaje complejo". Estas categorías son todas las psicosis crónicas no inducidas por sustancias, los trastornos graves del humor y los trastornos de personalidad paranoide, esquizoide, esquizotípico y límite. Además de estas categorías diagnósticas, se han incluido en el estudio otras que también producen una afectación prácticamente constante en el funcionamiento y que en su tratamiento requieren necesariamente tratamiento psiquiátrico tanto psicoterapéutico como farmacológico. Estas otras categorías añadidas incluyen determinados trastornos de ansiedad graves (agorafobias, fobias sociales generalizadas y trastorno obsesivo-compulsivo), los trastornos mentales orgánicos, los trastornos alimentarios, el trastorno antisocial de la personalidad y el trastorno por déficit de atención con hiperactividad (TDAH), siempre siguiendo los criterios internacionales de la clasificación CIE-10 (OMS, 1992). Así se ha establecido la definición de PDG (Luque Fuentes, 2001) y se ha estudiado al grupo de pacientes con este diagnóstico, analizándose, entre las variables estudiadas, aquellas que pueden determinar el tipo de alta recibida en nuestro centro.
En general, la literatura científica sobre patología dual no es concluyente y ofrece gran diversidad en los resultados. Generalmente los trabajos publicados se centran en el análisis de una sola sustancia o de un solo trastorno mental y no establecen la clasificación que hemos definido para categorizar el diagnóstico de patología dual grave, sin delimitar por tanto el tipo de patología dual estudiada.

Los primeros trabajos sobre adhesión al tratamiento en programas para drogodependientes, con pacientes ingresados en una comunidad terapéutica, fueron realizados a mediados y finales de los años setenta (Wexler y De Leon, 1977; Aron y Daily, 1976). Los resultados de estos estudios sugieren que los sujetos que abandonan suelen tener un problema más grave en cuanto al trastorno adictivo y la psicopatología asociada que los que permanecen y finalizan los tratamientos (Secades y Fernández-Hermida, 2000). La comorbilidad de la adicción con psicopatología previa (O’Brien, 1984; Arias, 2001) y con deterioro orgánico grave (Madoz-Gúrpide, Sais-Amorim, Baca-García y Ochoa, 2001) son también señalados como factores de mala respuesta al tratamiento en régimen de internamiento.

Por ello, se ha medido el cumplimiento del programa terapéutico relacionándolo con el tipo de alta (terapéutica versus abandono), determinado a su vez por la adhesión al tratamiento en un programa residencial libre de drogas. Se asume que el alta terapéutica indica el cumplimiento del programa completo con éxito, mientras que el alta no terapéutica se relaciona con el fracaso terapéutico, ya sea debida a abandono voluntario o expulsión.

\section{Metodología}

El presente estudio se ha llevado a cabo en una comunidad terapéutica profesional (CTP) de Marbella (Málaga), dedicada a la desintoxicación, tratamiento y posterior reinserción de pacientes adultos adictos, en régimen de internamiento. Se eligió este recurso por estar especializado en el diagnóstico y tratamiento de los trastornos mentales comórbidos, con una unidad psiquiátrica integrada en la propia CTP y en la que el mismo psiquiatra ha tratado toda la muestra, tanto en lo relativo al diagnóstico de TMG como de TUS, lo que incrementa la fiabilidad. Para el diagnóstico de PDG se han tenido en cuenta únicamente los TUS que cumplían criterios de dependencia según las clasificaciones internacionales DSM-IV-TR (American Psychiatric Association, 2002) y CIE-10 (OMS, 1992).

Las sustancias que se han incluido en este estudio son: alcohol, tabaco, opiáceos, cannabis, cocaína e hipnosedantes, realizando el estudio para cada paciente en todas aquellas para las cuales se cumplieran los criterios de dependencia. Consideramos "policonsumo" a la dependencia de varias sustancias, sin que se exista una sustancia principal de abuso.

En cuanto al concepto de enfermedad mental grave incluido en la PDG, se han seguido las pautas que define el 
Sistema Andaluz de Salud (SAS) en su mapa de procesos asistenciales (Consejería de Salud, 2006), con algunas modificaciones, como se ha señalado anteriormente.

El trabajo se ha realizado en una comunidad terapéutica profesional (CTP) con todos los recursos necesarios para la desintoxicación, el diagnóstico y el tratamiento integral de los pacientes duales graves incluyendo unidad psiquiátrica específica dentro del centro. Se ha considerado que es un buen medio para realizar esta investigación porque representa un modelo integrado, con un único equipo terapéutico competente para el abordaje de ambos trastornos. Los pacientes que ingresan en la CPT son evaluados a través de un modelo de entrevista clínica semi-estructurada elaborada en la propia CTP y de entrevistas de cribaje psiquiátrico MINI (Sheehan et al., 1998) y SCL-90-R (Derogatis y Savitz, 2000), por los psicólogos encargados de su seguimiento. Aquellos casos susceptibles de cumplir los criterios de Patología Dual Grave (PDG) según la modificación de las recomendaciones de la Junta de Andalucía, son reevaluados por un único psiquiatra, que en función de la entrevista clínica y los criterios de clasificación internacionales, diagnostica, confirma o desestima el diagnóstico previo. Estos pacientes tienen ciertas características de homogeneidad en cuanto a la gravedad, evaluación psiquiátrica y seguimiento, y en algunos casos han sido reevaluados y rediagnosticados tras ver la evolución de sus síntomas, cuando habían permanecido en el programa el tiempo suficiente para descartar sintomatología secundaria a intoxicación o abstinencia.

\section{Muestra}

El trabajo se ha realizado sobre los 805 pacientes adultos con trastornos por uso de sustancias (TUS) ingresados en la CTP "Hacienda de Toros" de Marbella entre junio de 2000 y junio de 2009, habiéndose descartado los ingresos de duración inferior a un mes por la insuficiente evaluación. De los 805 pacientes que fueron valorados, se seleccionaron la población objeto del estudio compuesta por 325 pacientes diagnosticados de PDG, 256 hombres y 69 mujeres. La edad media de inicio de la dependencia de sustancias es de 20,12 años (ds: 6,78) y la edad media al inicio del tratamiento es de 38,12 años (ds: 9,16). Las características de la muestra se definen en la tabla 1 .

\section{Diseño}

Se trata de un estudio retrospectivo y transversal sin grupo control, basado en el análisis detallado de la información recogida en un modelo de entrevista clínica semi-estructurada diseñado en la CTP, y los instrumentos estandarizado MINI (Sheehan et al., 1998) y SCL-90-R (Derogatis y Savitz, 2000).

\section{Análisis estadístico}

Se realiza el estudio descriptivo de la muestra, para el cual se presentan media, desviación estándar, porcentaje válido, valores mínimo y máximo. En la comparación de
Tabla 1

Características de la muestra con PMG ( $N=325)$

\begin{tabular}{llr}
\hline \multirow{2}{*}{ Sexo } & Hombres & $78,8 \%$ \\
& Mujeres & $21,2 \%$ \\
Nivel & Sin estudios & $31,1 \%$ \\
educativo & Estudios primarios o secundarios & $61,8 \%$ \\
& Estudios superiores & $7,1 \%$ \\
Actividad & Activos & $14,5 \%$ \\
laboral & Desempleados & $55,7 \%$ \\
& Baja laboral permanente & $29,8 \%$ \\
& Dependencia de alcohol & $61,8 \%$ \\
& Dependencia de tabaco & $81,8 \%$ \\
Dependencia & Dependencia de opiáceos & $43,1 \%$ \\
de sustancias & Dependencia de cannabis & $51,7 \%$ \\
& Dependencia de cocaína & $78,2 \%$ \\
& Dependencia de hipnosedantes & $25,5 \%$ \\
& Policonsumo (sin incluir tabacol & $84,5 \%$ \\
\hline
\end{tabular}

variables cuantitativas, para comprobar estadísticamente las diferencias significativas de medias, se ha realizado la prueba de la t de Student para muestras independientes. Para el análisis comparativo de las variables cualitativas se emplea el test de la chi-cuadrado de Pearson. Se elige la significación bilateral, considerándose significativos los valores estadísticos para $\mathrm{p}<0,05$.

Para analizar de qué variables depende el tipo de alta, se ha realizado regresión logística binaria: método backward, con la prueba de razón de verosimilitud.

\section{Resultados}

El 39,7\% de la población estudiada (n=325) recibió diagnóstico de PDG. Dichos diagnósticos se describen en la tabla 2. De estos 325 pacientes con PDG, se obtiene que el 29,5\% de la muestra recibió alta terapéutica, entendiendo como tal el cumplimiento del programa en su totalidad, en condi-

Tabla 2

Resultados de la muestra con PMG (N=325)

\begin{tabular}{llc}
\hline & Trastornos psicóticos & $30,5 \%$ \\
& Trastornos afectivos graves & $36,3 \%$ \\
$\begin{array}{l}\text { Comorbilidad } \\
\text { psiquiátrica }\end{array}$ & T. graves ansiedad & $16,3 \%$ \\
& T. orgánicos & $8,9 \%$ \\
& T. límite personalidad & $20,6 \%$ \\
& TDAH & $14,5 \%$ \\
Tipo de alta & Alta terapéutica & $29,5 \%$ \\
& No terapéutica & $70,5 \%$ \\
\hline
\end{tabular}


Tabla 3

Variables significativas: Porcentaje de los que reciben alta terapéutica

\begin{tabular}{|c|c|c|c|}
\hline \multirow{2}{*}{ Sexo } & Mujeres & $39,1 \%$ & \multirow{2}{*}{$=.049$} \\
\hline & Hombres & $27,0 \%$ & \\
\hline \multirow{3}{*}{ Nivel educativo } & Estudios superiores & $43,5 \%$ & \multirow{3}{*}{$=.020$} \\
\hline & Estudios primarios/secundarios & $32,8 \%$ & \\
\hline & Sin estudios & $19,8 \%$ & \\
\hline \multirow{2}{*}{ Dependencia de opiáceos } & Dependientes & $22,9 \%$ & \multirow{2}{*}{$=.022$} \\
\hline & No dependientes & $34,6 \%$ & \\
\hline \multirow{2}{*}{$\begin{array}{l}\text { Dependencia de múltiples sustancias } \\
\text { ("policonsumo") }\end{array}$} & Policonsumo & $28,0 \%$ & \multirow{2}{*}{$=.013$} \\
\hline & No policonsumo & $55,6 \%$ & \\
\hline \multirow{2}{*}{ PDG con trastornos psicóticos } & Trastornos psicóticos & $19,2 \%$ & \multirow{2}{*}{$=.007$} \\
\hline & No diagnosticados de psicosis & $34,1 \%$ & \\
\hline \multirow{2}{*}{ Trastorno límite de personalidad (TLM) } & TLM & $19,4 \%$ & \multirow{2}{*}{$=.041$} \\
\hline & No TLM & $32,2 \%$ & \\
\hline
\end{tabular}

Tabla 4

Variables cuantitativas y su significación en función del tipo de alta, sin ajustar

\begin{tabular}{lcccc}
\hline \multicolumn{4}{c}{ Tipo de alta (terapéutica vs No terapéutica) } \\
\hline & \multicolumn{4}{c}{ I.C. $95 \%$} \\
\cline { 2 - 5 } & $\begin{array}{c}\text { Dif de } \\
\text { medias }\end{array}$ & Inferior & Superior & Sig. \\
\hline $\begin{array}{l}\text { Edad al inicio de } \\
\text { tratamiento }\end{array}$ & 0,153 & $-2,202$ & 2,508 & 0,898 \\
Edad inicio consumo & $-1,554$ & $-3,359$ & 0,251 & 0,091 \\
Ingresos hospitalarios & 0,510 & 0,089 & 0,932 & 0,018 \\
Ingresos en CT & 0,476 & 0,071 & 0,881 & 0,021 \\
\hline
\end{tabular}

ciones de estabilización en cuanto al trastorno mental y en abstinencia absoluta de sustancias, continuando su evaluación posterior a nivel ambulatorio.

Del análisis bruto de variables cualitativas y cuantitativas, encontramos que en relación con el alta terapéutica, son significativas las siguientes variables: Sexo $(\mathrm{p}=0,049)$, así el porcentaje de mujeres que reciben alta terapéutica $(39,1 \%)$ es mayor que el de hombres $(27,0 \%)$. Nivel de estudios $(\mathrm{p}=0,020)$, así a mayor nivel de estudios, mayor porcentaje de alta terapéutica. En los pacientes con estudios superiores este porcentaje es del $43,5 \%$ frente al $32,8 \%$ en primarios/ secundarios y solo el $19,8 \%$ en los pacientes sin estudios. Dependencia de opiáceos $(\mathrm{p}=0,022)$, los dependientes de opiáceos tienen peor pronóstico con el 22,9\% de alta terapéutica, frente a los que no son dependientes de esta sustancia que recibieron alta terapéutica en el 34,6\% de los casos. La dependencia de múltiples sustancias ("policonsumo”) disminuye el porcentaje de alta terapéutica $(28,0 \%)$ frente a los que no la presentan $(55,6 \%)(p=0,013)$. Para PDG con trastornos psicóticos la frecuencia de alta terapéutica es menor $(19,2 \%)$ que en los pacientes no diagnosticados de psicosis $(34,1 \%)(p=0,007)$. Los pacientes con trastorno límite de personalidad reciben alta terapéutica en porcentaje mucho menor $(19,4 \%)$ que los que no lo padecen $(32,2 \%)$ $(\mathrm{p}=0,041)$ (Tabla 3, 4 y 5).

En el análisis de regresión logística (ajustado), para ver qué variables influyen en el alta terapéutica se han introducido las siguientes: sexo, estudios, situación laboral, edad de inicio del consumo, edad al inicio del tratamiento, dependencia de alcohol, tabaco, opiáceos, cannabis, cocaína, hipnosedantes, policonsumo, trastornos psicóticos, trastornos afectivos, trastorno límite de personalidad, otros trastornos de personalidad, TDAH, trastornos de ansiedad, otras patologías psiquiátricas (que incluye los trastornos mentales orgánicos y los trastornos alimentarios), y "policomorbilidad" psiquiátrica. De todas estas variables introducidas en la ecuación, se muestran significativas: sexo, edad al inicio del tratamiento, grado de estudios del paciente, dependencia de múltiples sustancias ("policonsumo"), trastornos psicóticos y trastorno límite de personalidad (Tabla 6).

Por cada año que aumenta la edad al inicio del estudio, disminuye la probabilidad de alta terapéutica 1,02 veces (IC 95\%: 1,000-1,060) ( $\mathrm{p}=.049)$.

El nivel de estudios aumenta la probabilidad de alta terapéutica. 2,017 veces en estudios primarios/secundarios frente a no tener estudios (IC95\%: 1,107-3,676) ( $\mathrm{p}=.022)$. Para los estudios superiores la probabilidad de alta terapéutica aumenta 4,015 veces frente a no tener estudios (IC 95\%: 1,393-11,571) $(\mathrm{p}=.010)$.

El policonsumo disminuye la probabilidad de alta terapéutica 4,329 veces (IC 95\%: 1,495-12,500) ( $\mathrm{p}=.007$ ) fren- 
Tabla 5

Variables cualitativas y su significación en función del tipo de alta, sin ajustar

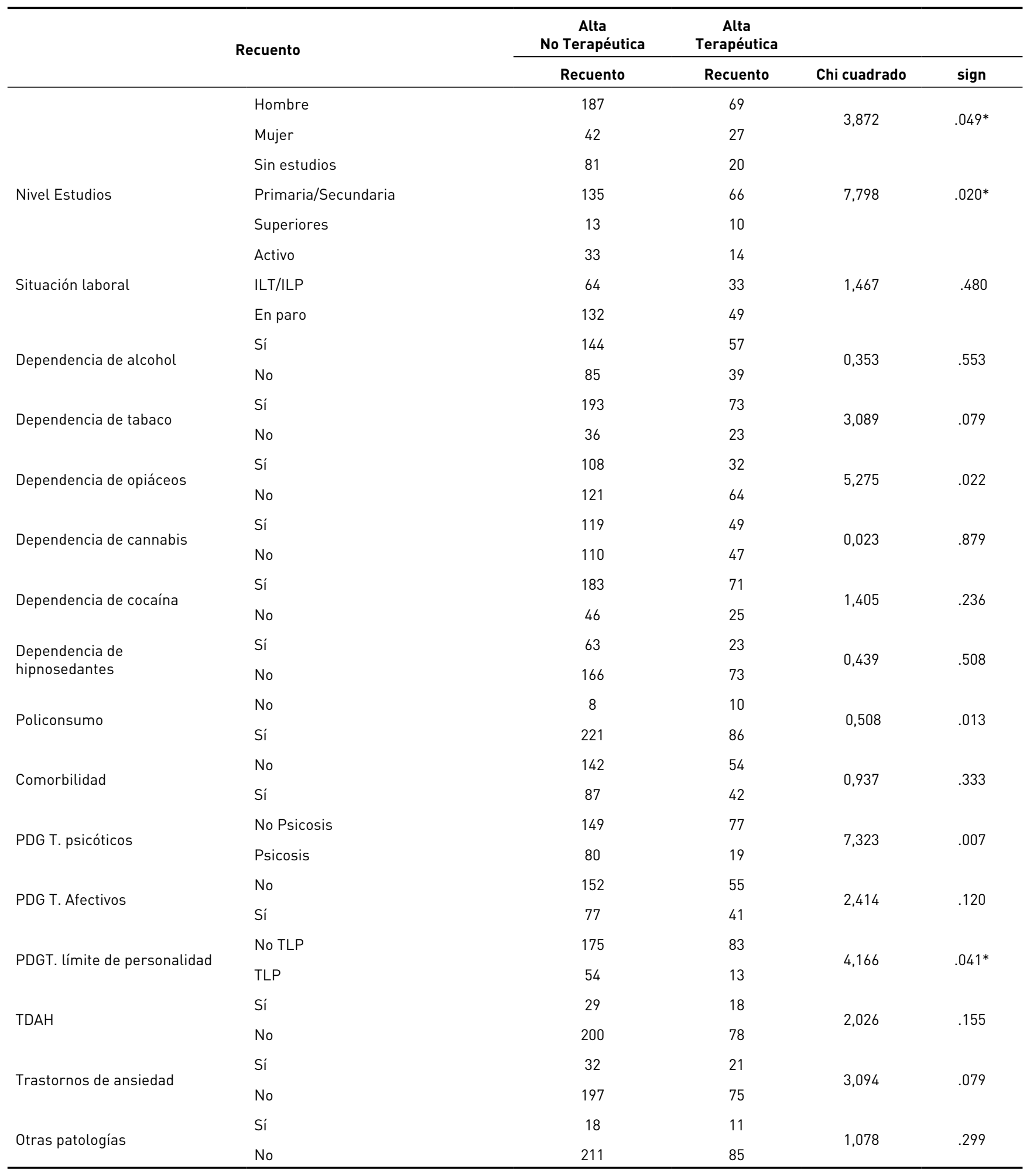

te al monoconsumo. Consumir más de una sustancia hace 4,329 veces menos probable el alta terapéutica que consumir sólo una.

La presencia de diagnóstico de trastorno psicótico disminuye la probabilidad de alta terapéutica 2,439 veces (IC 95\%: $1,326-4,484)(\mathrm{p}=.004)$ respecto a no tener ese diagnóstico.
El diagnóstico de trastorno límite de personalidad disminuye la probabilidad de alta terapéutica 3,115 veces (IC 95\%: $1,510-6,451)(\mathrm{p}=.002)$ frente a la no presencia de ese diagnóstico. 
Tabla 6

Variables significativas tras el ajuste

\begin{tabular}{|c|c|c|c|c|c|}
\hline & \multirow{2}{*}{ B } & \multirow{2}{*}{ Sig } & \multicolumn{3}{|c|}{ IC $95 \%$ para Exp(B) } \\
\hline & & & $\operatorname{Exp}(B)$ & Inferior & Superior \\
\hline Edad & $-0,030$ & .049 & 0,971 & 0,943 & 1,000 \\
\hline Estudios & & .015 & & & \\
\hline Estudios(1) & 0,702 & .022 & 2,017 & 1,107 & 3,676 \\
\hline Estudios(2) & 1,390 & .00 & 4,015 & 1,393 & 11,571 \\
\hline Policonsumo & $-1,466$ & .007 & 0,231 & 0,080 & 0,669 \\
\hline PDG-psicosis & $-0,892$ & .004 & 0,410 & 0,223 & 0,754 \\
\hline PDG -TLP & $-1,137$ & .002 & 0,321 & 0,155 & 0,662 \\
\hline Constante & 3,527 & .003 & 34,03 & & \\
\hline
\end{tabular}

Estudios (1): Primarios/secundarios vs no estudios; Estudios (2): Superiores vs no estudios; PDG: Patología dual grave; TLP: Trastorno límite personalidad

\section{Discusión}

En este trabajo se ha estudiado a un grupo de pacientes adultos adictos, con diagnóstico de PDG, analizando las variables que pueden condicionar el tipo de alta recibida en la comunidad terapéutica profesional "Hacienda de Toros", un recurso de adicciones muy especializado tanto para el tratamiento de dependencias de sustancias como para el abordaje terapéutico de los pacientes con TUS y trastornos mentales comórbidos, con una unidad psiquiátrica integrada en la propia CT. Para ello, se ha evaluado el alta terapéutica al final del programa.

Es un punto fuerte el tamaño muestral en relación a las hipótesis propuestas y a la cantidad de variables estudiadas. También la evaluación clínica en todos los casos por parte del mismo psiquiatra, en la caracterización y diagnóstico de la muestra estudiada, con las mismas herramientas, que incrementa la fiabilidad al no introducir distintos criterios. Uno de los esfuerzos más destacados y que caracteriza este estudio es el empleo de criterios bien definidos, reproducibles y fiables para clasificar y caracterizar la muestra. También se considera interesante no haber restringido el análisis a una sustancia única, sino haber tenido en cuenta todas las dependencias en casos de policonsumo, y todos los diagnósticos de trastorno mental grave en caso de comorbilidad psiquiátrica.

Por otra parte, el análisis se ha realizado en un dilatado periodo de tiempo, a lo largo de 9 años, en tanto que los ofrecidos por diferentes publicaciones consultadas suelen limitarse generalmente a periodos más cortos de tiempo. Pudieran ser limitaciones el propio hecho de realizar el trabajo en una unidad tan específica y de características particulares, que pueden hacer poco probable la comparación con otras muestras aunque existen dificultades añadidas debido a la escasa homogeneidad, en general, de los criterios empleados para caracterizar este tipo de muestras. En el presente trabajo no existe un grupo control, si bien es cierto que dado su diseño, existe una comparación entre cada submuestra con el total.
Los resultados encontrados en nuestro estudio en cuanto al número de altas terapéuticas en pacientes con PD coinciden con los de Lois y Álvarez (2010) que indican un 26,0\% para alta terapéutica en una muestra de 208 pacientes de una comunidad terapéutica. Los resultados de otro estudio anterior (López-Goñi, Fernández-Montalvo, Illescas, Landa y Lorea, 2008) encontraron un 32,8\% de alta terapéutica.

En cuanto al género, nivel de estudios, y policonsumo, las variables significativas coinciden con algunas halladas en estudios realizados otros contextos terapéuticos (Guerrero-Huesca, García-Aurrecoechea, Díaz-Negrete y Balanzario, 2001; Kelly, O’Grady, Mitchell, Brown y Scharwrtz, 2011). Hay una gran variabilidad en los resultados de los diferentes programas terapéuticos y así en un reciente estudio sobre tratamiento con metadona, se concluye que la tasa de retención en mujeres es más elevada que en los hombres y en el estudio de López-Goñi et al. (2008) la mayor parte de los abandonos definitivos (8 de cada 10) se producen entre los varones. Que el ser mujer suponga mejor pronóstico podría deberse, entre otros factores, a su menor gravedad de consumo y a un mejor apoyo psicosocial (Arnáez, Marina-González, Secades y Fernández-Hermida, 2004; Urbano-Aljama, 2006) aunque existe controversia sobre esta cuestión (Ochoa y Madoz-Gúrpide, 2008; Ochoa, Arias, Somoza y López-Ibor, 1992).

En el ya mencionado estudio de López Goñi et al. (2008) las personas que abandonan definitivamente el tratamiento son significativamente de mayor edad. Parece lógico que una mayor edad del paciente suponga una más extensa historia de consumos y fracasos terapéuticos previos, lo que apoya el peor pronóstico.

En el presente trabajo, los niveles mayores en estudios se relacionan con un mayor porcentaje de alta terapéutica. En el trabajo publicado sobre una muestra de 1432 pacientes ambulatorios en tratamiento con naltrexona (Ochoa y Madoz-Gúrpide, 2005), si bien el nivel educativo no guarda aparentemente relación con el pronóstico, sí parece hacerlo un nivel profesional superior. El estudio NTIES (1997) en- 
contró que los pacientes con reducciones más importantes en la gravedad del uso de drogas eran los que tenían un mayor nivel educativo cuando accedieron al tratamiento y además estos pacientes se caracterizaban por completar el tratamiento. Cabe pensar que niveles de estudios superiores suponen mejores estrategias de afrontamiento de la adicción y mayor probabilidad de trabajar adecuadamente con los contenidos de las terapias psicológicas.

En nuestro estudio, el policonsumo disminuye el porcentaje de alta terapéutica $(28,0 \%)$ frente a los que no son policonsumidores $(55,6 \%)$. En este sentido los resultados encontrados en otros estudios (Guerrero Huesca, GarcíaAurrecoechea, Díaz-Negrete y Balanzario, 2001; Rush, 1979) aunque alejados en el tiempo, y heterogéneos en cuanto a los datos, avalan esta tendencia ya señalada por otros autores (Ochoa, Arias, Somoza y López-Ibor, 1992) que incluye tanto el policonsumo como la severidad de la adicción. El policonsumo suele agravarse por la edad y se relaciona con mala alimentación, descenso de actividad física y disminución de intereses culturales, repercutiendo también directamente en la economía y la dinámica familiar social (Aizpiri et al., 2002).

Los dependientes de opiáceos tienen, en nuestro estudio, peor pronóstico de alta terapéutica, que los que no son dependientes de esta sustancia. Los tratamientos en dependencia de opiáceos más conocidos y utilizados actualmente en las comunidades terapéuticas son los tratamientos con antagonistas puros como la naltrexona (Ochoa y Madoz-Gúrpide, 2005) además de los tratamientos de deshabituación sin fármacos específicos y los programas de mantenimiento con metadona. Otro estudio (De Jong, Roozen, Van Rossum, Krabbe y Kerkhof, 2007) en dependientes de opiáceos, la eficacia de mantenimiento con naltrexona en combinación con el enfoque de refuerzo comunitario a los 10 meses de tratamiento alcanzó tasas de abstinencia del 28,0\%. La tasa de abstinencia acumulada en 16 meses fue del $24,0 \%$. El resultado encontrado en nuestra muestra es semejante a los citados estudios sobre dependientes de opiáceos. También coincide con otros trabajos (Madoz-Gúrpide, Ochoa, Salvador y Baca-García, 2003) donde el porcentaje de alta terapéutica fue del $24,0 \%$. Hay que considerar, no obstante, que los resultados y medidas empleadas en los estudios citados no son homogéneas, más bien al contrario. Se podría resumir que el consumo de opiáceos supone un peor pronóstico al menos en retención en tratamiento y tipo de alta, siendo un dato común a todos ellos.

Las dependencias de alcohol, cannabis, cocaína o hipnosedantes, no muestran diferencias significativas en el estudio del pronóstico en función del tipo de alta en nuestro trabajo. Sin embargo otros trabajos como el realizado por Crits-Christoph et al. (1999) sobre 487 individuos consumidores de cocaína en tratamiento encuentran que el porcentaje de abstinencia a los tres meses de iniciados los tratamientos era de $26.4 \%$. En otro estudio realizado por Sanchez-Hervás et al. (2010) sobre 91 pacientes con depen- dencia de cocaína en tres unidades de conductas adictivas y centros de tratamiento ambulatorio públicos, el porcentaje final de retención a los seis meses fue del 49,5\%. Dado que los resultados son heterogéneos, cabe pensar que dependen en gran medida no solo del tipo de sustancia que produzca la dependencia, sino del perfil psicopatológico de los pacientes, del centro de tratamiento, y del estadio personal en la motivación para el cambio en los patrones de conducta.

En los pacientes diagnosticados de trastornos psicóticos, la frecuencia de alta terapéutica es menor que en los pacientes no diagnosticados de psicosis. Desde hace décadas se conoce que el consumo repercute tanto en la clínica como en el curso de la enfermedad ocasionando que los pacientes duales con esquizofrenia tengan dificultades para la adhesión al tratamiento (Santamarina e Iglesias, 2001), un mayor incumplimiento terapéutico y más ingresos psiquiátricos (Bartels et al., 1993); inicio más temprano de la psicosis, mayor incidencia de problemas legales y desempleo, refractariedad relativa al tratamiento neuroléptico, y mayor frecuencia de recaídas y suicidios (Ortiz-Lobo, 1998). Así, los pacientes con patología dual que padecen esquizofrenia tienen un peor pronóstico general (Arias, Sánchez y Padín, 2002).

Los pacientes con trastorno límite de personalidad reciben alta terapéutica en porcentaje menor frente a los que no lo padecen. Los trastornos de personalidad no han sido, sin embargo, muy estudiados en las comunidades terapéuticas (Fernández-Montalvo et al., 2004), aunque un indeterminado pero numeroso grupo de pacientes con este trastorno recibe tratamientos en recursos para drogodependencias (Gunderson, 2001) y en general la presencia de un trastorno de personalidad se asocia con una menor adhesión al tratamiento (Hunter et al., 2000; Wölver, Burtscheid, Redner, Schwartz y Gaebel, 2001) y con el abandono prematuro del mismo aunque algunos autores (Linehan et al., 2006) ofrecen resultados esperanzadores respecto a la eficacia de la terapia dialéctico-comportamental. Un metaanálisis sobre 41 estudios en Trastorno Límite (Barnicot, Katsakou, Marougka y Priebe, 2011) obtienen cifras muy variables de cumplimiento terapéutico, que van desde el $36,0 \%$ al $100,0 \%$, con una tasa global del $71,0 \%$. Al ser un trastorno que hasta ahora no tiene un tratamiento farmacológico específico, el abandono voluntario podría deberse al "síntoma impulsividad" asociado, que no siempre es bien controlado por las terapias con fármacos actuales, aunque mejora cuando el tiempo de estancia en comunidad terapéutica es prolongado (Bankston et al., 2009). Otra posible explicación es la dificultad que estos pacientes tienen al sometimiento a las normas y en el manejo de las relaciones interpersonales grupales, lo que podría facilitar el abandono del programa terapéutico.

En vista de los resultados obtenidos, consideramos que este tipo de estudios deberían ser continuados con un seguimiento prolongado en el tiempo sobre la evolución de los diferentes marcadores de éxito terapéutico (tiempo de abstinencia, medidas de adaptación social...). Sería deseable 
plantearse la homogeneización de criterios de clasificación tanto en lo concerniente a los trastornos por uso de sustancias, como en la propia definición de patología dual grave. Consideramos prioritaria la importancia de las variables predictoras de pronóstico terapéutico, tomándolas como referencia a la hora de planificar los tratamientos. Cabe señalar la conveniencia de individualizar al máximo los programas terapéuticos adaptándolos a cada variable, y no al revés, pues son las variables individuales las que marcan el pronóstico.

\section{Conclusiones}

Los resultados principales coinciden con publicaciones previas y no parecen, por otra parte, diferir sustancialmente de los resultados obtenidos en otras modalidades terapéuticas diferentes de las CCTT. Así, en nuestro estudio, el género condiciona el tipo de alta, encontrándose mayores porcentajes de alta terapéutica para las mujeres. El mayor nivel de estudios incrementa también el mejor pronóstico entendido como mayor frecuencia de alta terapéutica en individuos con estudios superiores. La edad más tardía al inicio del tratamiento disminuye la probabilidad de alta terapéutica. Del mismo modo, el policonsumo, el diagnóstico de trastornos psicóticos y de trastorno límite de personalidad se relacionan con peores resultados obteniéndose menores porcentajes de altas terapéuticas. Reconocer estas características permitirá la identificación precoz de los pacientes que tienen más riesgo de abandonar precipitadamente el tratamiento, para tratar de prevenirlo aumentando la intensidad terapéutica.

\section{Conflicto de intereses}

Los autores del artículo manifiestan no tener ningún conflicto de intereses.

\section{Referencias}

Aizpiri, J., Barbado, J. A., Cañones, P. J., Fernández-Camacho, A., Gonçalves, F., Rodríguez-Sendín, J.,.... Solla-Camino J. M. (2002). Trastornos por sustancias de abuso (y III). Medicina General, 48, 814-823.

American Psychiatric Association. (2002). DSM-IV-TR. Manual diagnóstico y estadístico de los trastornos mentales. Texto revisado. Barcelona: Masson.

Arias, F. (2001). Comorbilidad de trastornos psiquiátrico y trastornos adictivos. En: Ochoa Mangado E. Antagonistas opiáceos en las dependencias. Clínica de la Naltrexona. Col. Psiquiatría 21. Barcelona: Ed. Ars Medica. Psiquiatría Editores SL, 105-125.

Arias, F., Sánchez, S. y Padín, J. J. (2002). Consumo de drogas y riesgo de recaída en la esquizofrenia. Psiquiatría Biológica, 9, 63-67.

Arnáez, C., Marina-González, P. A., Secades, R. y Fernández-Hermida, J. R. (2004). Calidad asistencial y satisfac- ción de las mujeres en tratamiento por drogodependencia en Asturias. Adicciones, 16, 81-90.

Aron, W. S. y Daily, D. W. (1976). Graduates and splitees from therapeutic community drug treatment programs: A comparison. International Journal of the Addictions, 11, 1-18.

Bankston, S. M., Carroll, D. D., Cron, S. G., Granmayeh, L. K., Marcus, M. T., Moeller, F. G.,... Liehr, P. R. (2009). Substance abuser impulsivity decreases with a nine-month stay in a therapeutic community. American Journal Drug and Alcohol Abuse, 35, 417-420.

Barnicot, K., Katsakou, C., Marougka, S., Priebe, S. (2011). Treatment completion in psychotherapy for borderline personality disorder - a systematic review and meta-analysis. Acta Psychiatrica Scandinavica, 123, 327-338.

Bartels, S. J., Teague, G. B., Drake, R. E., Clark, R. E., Bush, P. W. y Noordsy, D. L. (1993). Substance abuse in schizophrenia: Service utilization and costs. Journal of Nervous and Mental Disease, 181, 227-232.

Chambers, R. A., Cristal, J. H. y Self, D. W. (2001). A neurobiological basis for substance abuse comorbidity in schizophrenia. Biological Psychiatry, 50, 71-83.

Compton, W. M., Thomas, Y. F., Stinson, F. S. y Grant, B. F. (2007). Prevalence, correlates, disability, and comorbidity of DSM-IV. Drug Abuse and Dependence in the United States. Results from the National Epidemiologic Survey on Alcohol and Related Conditions. Archives of General Psychiatry, 64, 566-576.

Consejería de Salud (2006). Trastorno Mental Grave: proceso asistencial integrado. En: Guía de diseño y mejora continúa de procesos asistenciales. Plan de calidad. Junta de Andalucía. Sevilla.

Crits-Christoph, P., Siqueland, L., Blaine, J., Frank, A., Luborsky, L., Onken, L. S.,.... Beck, A. T. (1999). Psychosocial treatments for cocaine dependence. National Institute on Drug Abuse Collaborative Cocaine Treatment Study. Archives of General Psychiatry, 56, 493-501.

De Jong, C. A., Roozen, H. G., Van Rossum, L. G., Krabbe, P. F. y Kerkhof, A. J. (2007). High Abstinence Rates in Heroin Addicts by a New Comprehensive Treatment Approach. American Journal on Addictions, 16, 124-130.

Derogatis, L. R. y Savitz, K. L. (2000). The SCL-90-R and Brief Symptom Inventory (BSI) in primary care. In M. E. Maruish (Ed.), Handbook of psychological assessment in primary care setting. Mahwah: Lawrence Erlbaum, 297-334.

Fernández-Montalvo, J., López-Goñi, J. J., Landa, N., Illescas, C., Lorea, I. y Zarzuela, A. (2004). Trastornos de personalidad y abandonos terapéuticos en pacientes adictos: resultados en una comunidad terapéutica. International Journal of Clinical and Health Psychology, 4, 271-283.

Galbaud, G., Newman, S. C. y Bland, R. C. (1993). Psychiatric Comorbidity and treatment seeking. Sources of selection bias in the study of Clinical population.Journal of Nervous and Mental Disease, 181, 467-474. 
Guerrero Huesca, J. A., García-Aurrecoechea, V. R., Díaz-Negrete, D. B. y Balanzario, M. C. (2001). Adherencia a tratamiento en una muestra de usuarios de drogas en Centros de Integración Juvenil. Dirección de Prevención, Subdirección de Investigación, Informe de Investigación 99-09. México.

Gunderson, J. G. (2001). Borderline Personality disorder. A Clinical guide. Washington: American Psychiatric Press.

Hunter, E., Powell, B., Penick, E., Nickel, J., Liskow, B., Centrell, P. y Landon, J. (2000). Comorbid psychiatric diagnosis and long-term drinking outcome. Comprehensive Psychiatry, 41, 334-338.

Kelly, S. M., O’Grady, K. E., Mitchell, S. G., Brown, B. S. y Scharwrtz, R. P. (2011). Predictors of methadone treatment retention from a multi site study. A survival analysis. Drug and Alcohol Dependence, 117, 170-175.

Kessler, R. C., McGonagle, K. A., Zhao, S., Nelson, C. B., Higes, M., Eshleman, S., Kendler, K. S. (1994). Lifetime and 12-month prevalence of DSM-III-R psychiatric disorders in the United States. Results from the National Comorbidity Survey. Archives of General Psychiatry, 51, 8-19.

Linehan, M., Comtois, K., Murray, A., Brown, M., Gallop, R., Heard, H., .... Lindenboim, N. (2006). Two-Year Randomized Controlled Trial and Follow-up of Dialectical Behavior Therapy vs Therapy by Experts for Suicidal Behaviors and Borderline Personality Disorder. Archives of General Psychiatry, 63, 757-766.

Lois, E. y Álvarez, J. A. (2010). Motivación y retención en un tratamiento de adicción a la cocaína. $11^{\circ}$ Congreso Virtual de Psiquiatría. Interpsiquis. Disponible en www.interpsiquis.com Febrero-Marzo. Psiquiatria.com

López-Goñi, J. J., Fernández-Montalvo, J., Illescas, C., Landa, N. y Lorea, I. (2008). Razones para el abandono del tratamiento en una comunidad terapéutica. Trastornos Adictivos, 10, 104-111.

Luque Fuentes, E. (2001). Patología dual grave en una comunidad terapéutica profesional dotada de unidad psiquiátrica (tesis doctoral ) UGR 2011-10-27.

Madoz-Gúrpide, A., Ochoa, E., Salvador, E. y Baca-García, E. (2003). Definición del perfil de indicación de los programas de naltrexona en la adicción a opiáceos: aplicación clínica de las técnicas de análisis de supervivencia. Adicciones, 15, 57-76.

Madoz-Gúrpide, A., Sais-Amorim, A., Baca-García, E. y Ochoa, E. (2001). Aspectos criminológicos en pacientes adictos a heroína: Relación entre toxicomanía y delincuencia. Actas Españolas Psiquiatría, 29, 221-227.

NTIES. National Treatment Improvement Evaluation Study. Center For Substance Abuse Treatment (1997). The Final Report. Disponible en URL: www.icpsr.umich. edu/SAMHDA/NTIES/NTIES-PDF/ ntiesfnl.pdf.

O'Brien, C. P. (1984). Trexan in opioid addiction. A comprehensive Product Monograph, 1-54.
Ochoa, E. y Madoz-Gúrpide, A. (2005). Situación actual de los programas con naltrexona en la dependencia de opiáceos. Revisión de su efectividad. Adicciones, 17, 226229.

Ochoa, E. y Madoz-Gúrpide, A. (2008). Diferencias de género en el tratamiento de la dependencia de opiáceos con naltrexona. Actas Españolas Psiquiatría, 36, 197-204.

Ochoa, E., Arias, F., Somoza, J. C. y López-Ibor, J. J. (1992). Tratamiento con naltrexona en dependientes de opiáceos: dos años y medio de seguimiento. Archivos Neurobiología, 55, 224-227.

OMS (1992). CIE-10: Trastornos Mentales y del Comportamiento: Descripciones clínicas y pautas para el diagnóstico. Madrid: Meditor.

Ortiz-Lobo, A. (1998). Esquizofrenia y consumo de drogas. Psiquiatría Pública, 10, 372.

Regier, D. A., Farmer, M. A., Rae, D. S., Locke, B. Z., Keith, S. J., Judd, L. L. y Goodwin, F. K. (1990). Comorbidity of mental disorder with alcohol and other drug abuse. Results from the epidemiologic catchment area (ECA) study. Journal of American Medical Association, 264, 2511-2518.

Rush, T. V. (1979). Predicting treatment outcomes for juvenile and young-adult clients in the Pennsylvania substance-abuse system. En: Beschner, G. M. y Friedman, A. S. Eds. Youth drug abuse: problems, issues and treatment. Lexington, M. A: Lexington Books.

Sánchez-Hervás, E., Secades, R., Santonja, F. J., Zacarés, F., García-Rodríguez, O., Martín-Yánez, E.,....García-Fernández, G. (2010). Abandono del tratamiento en adictos a la cocaína. Adicciones, 22, 59-64.

Santamarina, S. y Iglesias, C. (2001). Esquizofrenia y sustancias psicotropas de consumo frecuente en nuestro medio. Adicciones, 13, 385-391.

Secades, R. y Fernández-Hermida, J. R. (2000). Cómo mejorar las tasas de retención en los tratamientos de drogodependencias. Adicciones, 12, 356.

Sheehan, D. V., Lecrubier, Y., Sheehan, K, H., Amorin, P., Janavs, J., Weiller, E.,.... Dunbar, G. C. (1998). The Mini-International Neuropsychiatric Interview (M.I.N.I.): the development and validation of a structured diagnostic psychiatric interview for DSM-IV and ICD-10. Journal Clinical Psychiatry, 59, Suppl 20, 22-33.

Sobradiel, N. y García-Vicent, V. (2007). Consumo de tabaco y patología psiquiátrica. Trastornos Adictivos, 9, 30-36.

Urbano Aljama, A. (2006). El uso diferencial, según la variable género, de las herramientas terapéuticas en el tratamiento residencial. Trastornos Adictivos, 8, 276-281.

Wexler, H. K. y De Leon, G. (1977). The Therapeutic Community: Multivariate Prediction of Retention. American Journal of Drug and Alcohol Abuse, 4, 145-151.

Wölver, W., Burtscheid, W., Redner, C., Schwartz, R. y Gaebel, W. (2001). Out-patient behaviour therapy in alcoholism: Impact of personality disorders and cognitive impairments. Acta Psychiatrica Scandinavica, 103, 30-37. 